\title{
Diagnostic performance of brain computed tomography to detect facial bone fractures
}

\author{
Duk Ho Kim ${ }^{1}$, Yoon Hee Choi ${ }^{1}$, Seong Jong Yun², Sun Hwa Lee \\ 'Department of Emergency Medicine, Ewha Womans University Hospital, Seoul, Korea \\ ${ }^{2}$ Department of Radiology, Aerospace Medical Center, Republic of Korea Air Force, Cheongwon, Korea \\ ${ }^{3}$ Department of Emergency Medicine, Sanggye Paik Hospital, Inje University College of Medicine, Seoul, \\ Korea
}

Objective Most patients with head trauma require brain computed tomography (CT) in the emergency department. However, the requirement for facial CT remains controversial. The aim of this study was to evaluate the diagnostic value of brain CT alone for detection of facial fractures and its ability to determine the requirement for additional facial CT.

Methods This retrospective multicenter study was conducted in two tertiary hospitals in Seoul, Republic of Korea, between January 2014 and December 2015. Data were collected from the medical records of adult patients (aged over 18 years) who had undergone both brain and facial CT on the same day as their presentation to the emergency department with blunt trauma to the head and face. The same radiologist analyzed all brain and facial CT images.

Results Eight hundred and sixty patients (668 men, 192 women; mean age 48.60 18.2 years) were identified to have had facial fractures. There was a statistically significant predominance of men but not of any particular age group. The sensitivity, specificity, positive predictive value, negative predictive value, and accuracy of brain CT were 83.72\%, 98.87\%, 97.17\%, 92.92\%, and $94.08 \%$, respectively.

Conclusion These findings suggest that brain CT has high diagnostic value for detection of upper facial bone fractures with high accuracy and can aid emergency physicians when determining the requirement for additional facial CT.

Keywords Tomography; Diagnosis; Facial bones; Skull

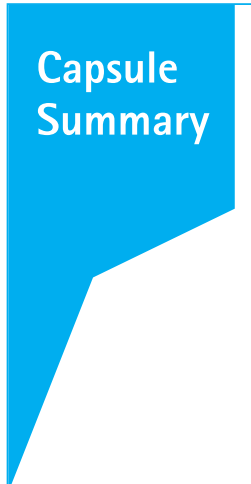

What is already known

The majority of patients with head trauma require brain computed tomography (CT) in the general emergency department. However, the requirement of facial CT remains controversial.

What is new in the current study

The results of our study suggest that brain CT has high diagnostic value for the detection of facial fractures with high accuracy and can aid emergency physicians in determining the requirement for additional facial CT.
Received: 23 March 2017

Revised: 24 June 2017

Accepted: 10 July 2017

Correspondence to: Sun Hwa Lee Department of Emergency Medicine, Sanggye Paik Hospital, Inje University College of Medicine, 1342 Dongil-ro, Nowon-gu, Seoul 01757, Korea E-mail:sunhwa9@hanmail.net

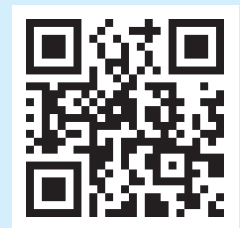

How to cite this article:

Kim DH, Choi YH, Yun SJ, Lee SH. Diagnostic performance of brain computed tomography to detect facial bone fractures. Clin Exp Emerg Med 2018;5(2):107-112.

This is an Open Access article distributed under the terms of the Creative Commons Attribution Non-Commercial License (http:// creativecommons.org/licenses/by-nc/4.0/). 


\section{INTRODUCTION}

The epidemiology and incidence of general emergency department (ED) admissions varies widely both nationally and internationally, largely because of differences in socioeconomic status and the regulation and consumption of alcohol. ${ }^{1}$ Regardless of these regional variations, the majority of patients with head trauma require brain computed tomography (CT) in the ED. However, the requirement for facial $\mathrm{CT}$ remains controversial.

Brain hemorrhage and facial fracture in patients with blunt trauma can be diagnosed by several methods. Although a thorough physical examination and careful assessment of history can aid in the decision to perform facial $\mathrm{CT}_{\text {, there are several limita- }}$ tions to these examinations, including requirement for intubation, unresponsiveness in certain circumstances, presence of equipment to protect the cervical spine, and language barriers. ${ }^{2}$

There have been remarkable developments in diagnostic imaging, so plain radiography of the face is rarely performed now because of its low sensitivity and specificity for detecting facial bone fractures. Together, brain and facial CT are considered to provide a rapid and precise diagnosis. ${ }^{3}$ However, emergency physicians also need to take into account the cost-effectiveness and radiation hazard of these procedures.

Previous studies have reported that the presence and location of facial soft tissue injuries may require both facial and brain $\mathrm{CT}^{4,5}$ However, a few studies have reported that brain CT may be adequate for screening of facial fractures, except for those affecting the nasal bone. ${ }^{6,7}$ The aim of this study was to evaluate the diagnostic value of brain $\mathrm{CT}$ alone for detection of facial fractures and its ability to determine the need for additional facial CT.

\section{METHODS}

\section{Study design and setting}

This retrospective study was conducted in two tertiary hospitals in Seoul, Republic of Korea. We assessed approximately 75,000 and 60,000 emergency visits to these two centers between January 2014 and December 2015 to identify patients older than 18 years who had undergone both brain and facial CT on the day of admission to the ED for blunt trauma to the head and face. At both centers, brain $\mathrm{CT}$ images were obtained in parallel with a tilted angle from the inferior orbital wall to the $\mathrm{C} 1$ vertebra and a collimation of $5 \mathrm{~mm}$. Facial CT was performed from the mandible to the vertex in the supine position with a collimation of $3 \mathrm{~mm}$.

This study was approved by the institutional review board of the Sanggye Paik Hospital, Inje University (2016-04-019-003).
This is a retrospective study based on electronic medical records of patients in the ED; thus, informed consent was waived.

\section{Data collection}

The following data were collected from the medical records by two blinded emergency physicians: patient age and sex; final diagnosis; presence of other injuries, such as facial lacerations, skin defects, lacrimal duct injuries, or eyeball injuries; location of the facial fracture, including the superior orbit, inferior orbit, medial orbit, nasal bone, and zygomatic arch; radiation dose; cost of each CT scan; disposition from ED; and findings at follow-up visits. Patients with mandibular fractures that could not be detected on brain CT were excluded.

\section{Reporting of outcome measures}

Before embarking on this study, we performed a preliminary pilot study to compare the accuracy of CT results between emergency physicians and radiologists and found no statistically significant differences between the two groups. However, to avoid discrepancies in reporting in the main study, both brain and facial CT images were analyzed by one radiologist blinded to the radiology reports and final clinical diagnoses to confirm the presence or absence of an acute facial fracture.

\section{Statistical analysis}

All statistical analyses were performed using PASW Statistics ver. 18.0 (SPSS Inc., Chicago, IL, USA). A P-value $<0.05$ was considered to be statistically significant. Fisher exact test and chi-square test were used to determine the sensitivity, specificity, positive predictive value, negative predictive value, and accuracy of brain CT in detecting facial fractures.

\section{RESULTS}

Of 2,900 patients identified to have undergone both brain and facial CT on the day of admission to the ED for blunt trauma to the head and face, 181 with mandibular fractures that could not be detected on brain CT were excluded. A diagnosis of brain hem-

Table 1. Baseline characteristics of patients with and without facial fractures included in the study

\begin{tabular}{lcccc}
\hline & Total & $\begin{array}{c}\text { Facial fracture } \\
\text { group }\end{array}$ & $\begin{array}{c}\text { No facial } \\
\text { fracture group }\end{array}$ & P-value \\
\hline Sex & & & & $<0.001$ \\
Male & 1,830 & 668 & 1,162 & \\
Female & 889 & 192 & 697 & \\
Age (yr) & $49.21 \pm 19.1$ & $48.60 \pm 18.2$ & $49.51 \pm 19.5$ & 0.248 \\
\hline
\end{tabular}


orrhage was recorded in 31 of these 181 patients, but no mortality and morbidity data were available for the other 150 patients. Finally, data for 2,719 patients were available for analysis. The baseline patient characteristics are shown in Table 1. The study population comprised 1,830 (69.4\%) men and 889 (30.6\%) women with a mean age of $49.21 \pm 19.1$ years. In total, 860 patients (668 men, 192 women; mean age $48.60 \pm 18.2$ years) had facial

Table 2. Sensitivity and specificity of brain CT for detection of facial fractures

\begin{tabular}{llcc}
\hline & & \multicolumn{2}{c}{ Facial CT } \\
\cline { 3 - 4 } & & No fracture & Fracture \\
\hline Brain CT & No fracture & $1,838(98.87 \%)^{\text {a) }}$ & 140 \\
& Fracture & 21 & $720(83.72 \%)^{\text {b) }}$ \\
\hline
\end{tabular}

$\mathrm{CT}$, computed tomography.

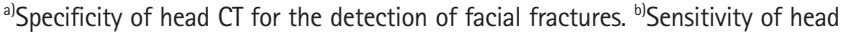
CT for the detection of facial fractures.
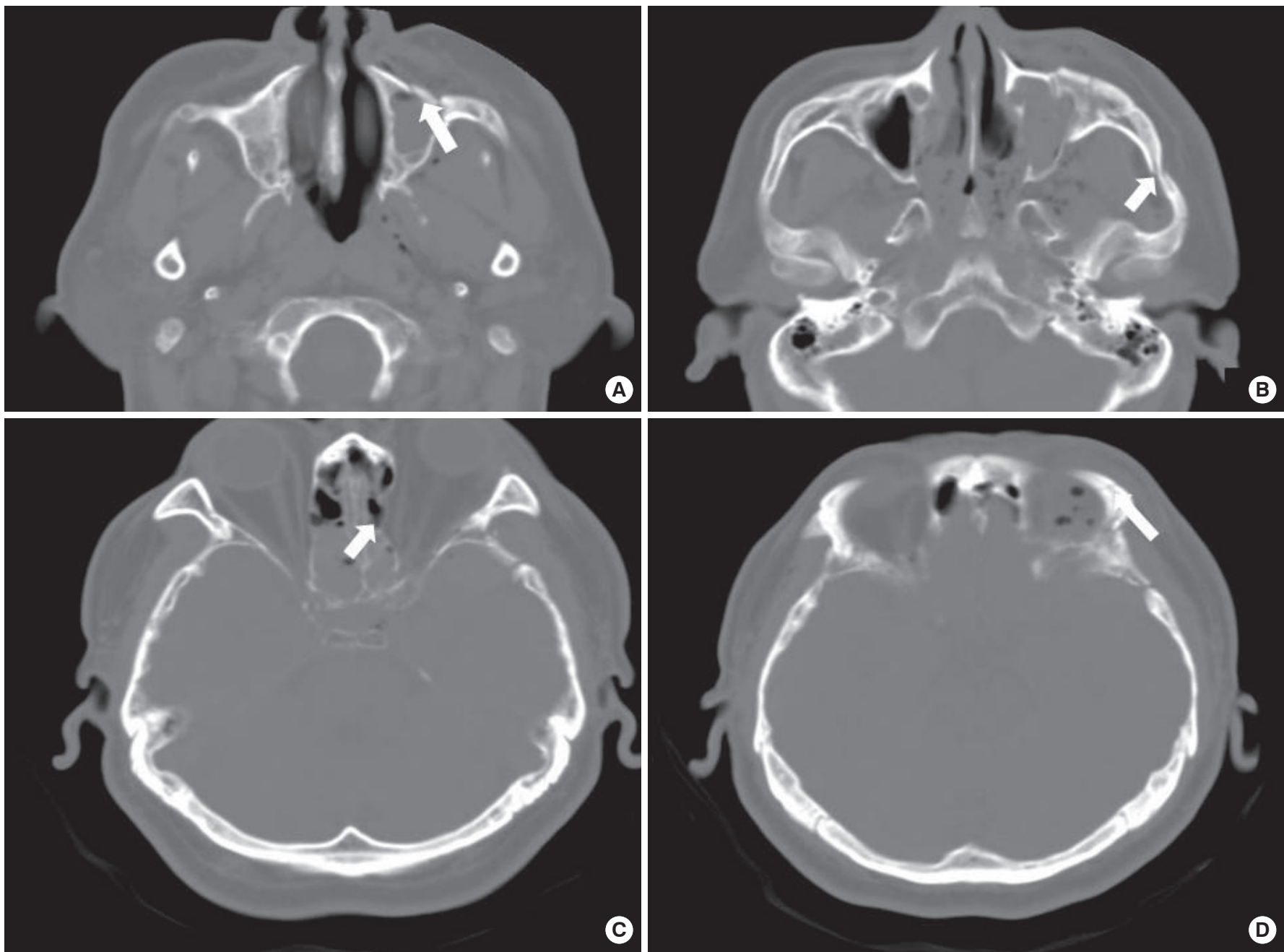

fractures. The remaining 1,859 patients (1,161 men, 697 women; mean age $49.51 \pm 19.5$ years) did not have facial fractures. There was a statistically significant predominance of men with facial fractures but not of any particular age group.

Table 3. Facial fractures missed on brain computed tomography

\begin{tabular}{lc}
\hline Facial fracture type & No. of fractures (\%) \\
\hline Medial orbital wall fracture & $14(8.9)$ \\
Lateral orbital wall fracture & $3(1.9)$ \\
Inferior orbital wall fracture & $18(11.5)$ \\
Superior orbital wall fracture & $9(5.7)$ \\
Nasal bone fracture & $90(57.3)$ \\
Zygomatic arch fracture & $7(4.5)$ \\
Maxillary fracture & $16(10.2)$ \\
Temporal bone fracture & $0(0)$ \\
Frontal bone fracture & $0(0)$ \\
Total & $157(100)$
\end{tabular}

Fig. 1. Brain computed tomography performed to detect facial fractures in a 54-year-old man. The arrows indicate fractures. Non-enhanced brain computed tomography images show fractures of (A) the maxilla, (B) the zygomatic arch, (C) the medial orbital wall, and (D) the superior orbital wall. 
The sensitivity and specificity of brain CT for detection of facial fractures is shown in Table 2. The sensitivity, specificity, positive predictive value, negative predictive value, and accuracy were 83.72\%, 98.87\%, 97.17\%, 92.92\%, and 94.08\%, respectively, indicating that brain CT had relatively good diagnostic accuracy for facial fractures (Fig. 1).

The most common facial fracture site was the nasal bone, followed by the medial and inferior orbital walls. In total, 483 patients required facial surgery. Brain CT could not detect 157 facial fractures in 140 patients. These fractures included 90 nasal bone fractures (57.3\%), 18 inferior orbit wall fractures (11.5\%), 16 maxillary fractures (10.2\%), and 14 medial orbital wall fractures (8.9\%) (Table 3). Of these fractures, only 15 involving the nasal bone required surgery. All temporal bone fractures and frontal bone fractures were detected on brain CT.

The mean effective radiation dose for brain CT and facial CT was 2.6 and $0.7 \mathrm{mSv}$, respectively. There was only a slight difference in the costs of brain CT and facial CT between the two hospitals, with brain CT costing approximately 100 US dollars with medical insurance coverage and 250 US dollars without insurance coverage and facial CT costing approximately 150 and 350 US dollars, respectively.

\section{DISCUSSION}

In most EDs, patients with blunt head and face trauma routinely undergo both brain and facial CT. $^{8}$ In patients with multiple trauma sites, brain, chest, abdominal, and extremity injuries are prioritized to rule out potentially life-threatening events. Facial injuries that do not compromise the airway are often neglected, potentially leading to permanent deformity and disability. ${ }^{9}$ Early recognition of a facial fracture requires both facial $\mathrm{CT}$ and brain $\mathrm{CT}$, so that prompt management can be implemented to improve outcomes.

Although radiation doses in conventional radiography for the head are lower than those in brain $\mathrm{CT}_{1}^{10}$ the interpretation of conventional radiographs is somewhat difficult for non-radiologists due to the complexity of the anatomy. ${ }^{11}$ Furthermore, conventional radiography for facial fractures has a lower diagnostic utility as compared with facial $\mathrm{CT}^{12,13}$ These might be the reasons for the missed fractures and delayed treatment in patients with facial trauma, which in turn increase the need for re-operation, increase the duration of ICU stay, increase medical costs, and result in poor cosmetic and functional outcomes. ${ }^{14}$ Thus, we propose brain CT as the screening method for facial fractures. Panoramic radiographs may be needed in patients with suspected mandibular fracture because brain $\mathrm{CT}$ does not cover the mandible.
When a facial fracture is strongly suspected on physical examination, particularly in patients with soft tissue lacerations and extensive swelling, facial CT is considered the most accurate method for detection of fractures. ${ }^{12,13,15}$ In the present study, we found that brain CT could detect facial fractures not involving the mandible with high specificity (98.87\%) and accuracy (94.08\%). These findings can aid in determining the requirement for additional facial CT scans.

Fractures of the nasal bone are the facial fractures most often undetected. It is speculated that brain CT is more reliable for detecting upper facial injuries than lower facial injuries. Nasal bone fractures can be diagnosed easily on physical examination, and a clinical diagnosis based on deformity and swelling is superior to radiographic confirmation, particularly for isolated nasal bone fractures. Therefore, we believe that brain CT has no clinical benefit in terms of the decision to acquire additional facial CT scans as far as nasal bone fractures are concerned. We recommend that when patients present with upper facial injuries, particularly those above the inferior orbital wall, brain CT should be performed first. If additional lesions are suspected and do not appear on brain $\mathrm{CT}_{\text {, }}$ facial CT should be performed.

Of the 157 fractures in 140 patients with facial fractures not detected on brain CT in our study, only 15 involving the nasal bone required surgery. Unlike other facial fractures, including those of the orbital walls, maxilla, and zygomatic arch, surgery for a nasal bone fracture is only required for cosmetic reasons. Therefore, nasal tip fractures would not have influenced the treatment plan in the present study. Other considerations with additional facial CT include the increased radiation exposure, cost, and time.

In Korea, the average costs of brain and facial CT are 100 and 150 US dollars, respectively. In the present study, we found 1,838 true-negative cases, meaning that neither brain CT nor facial CT detected facial fractures in these patients. The total cost after multiplying the average cost by 1,838 would be approximately 300 million dollars per year.

Following the atomic bombing of Japan in 1945, several studies on the survivors have been published, particularly with regard to radiation-induced cancer. ${ }^{16} \mathrm{CT}$ is now receiving increased attention as a major source of radiation exposure. ${ }^{17}$ The most important factor affecting the radiation dose is the number of scans. Other factors include the scanning duration, axial scan range, patient size, and pitch value. ${ }^{18}$

In the US, there has been a sharp increase in the number of CT procedures performed each year, from 3 million in the 1980s to 62 million at present. ${ }^{19}$ Acquisition of repeated CT scans or multiple CT scans for defensive medical treatment can lead to serious health problems and needs to be minimized. ${ }^{20}$ 
The radiation doses from CT procedures average between 0.7 and $30 \mathrm{mSv}$ depending on the organ being scanned. ${ }^{21}$ In one largescale study involving nuclear industry workers, it was reported that exposure to an average dose of approximately $20 \mathrm{mSv}$ for a single CT scan was associated with increased cancer mortality. ${ }^{22}$ In the present study, the mean effective radiation dose with brain CT was $2.6 \mathrm{mSv}$ and that with facial CT was $0.7 \mathrm{mSv}$. Therefore, the radiation dose could be decreased by approximately $21 \%$ for each patient if only brain CT was performed.

This study has several limitations. First, our retrospective review of patient data could have resulted in a certain degree of reporting bias because of missing and incomplete charts. Second, mandibular fractures were excluded because they could not be detected on brain $\mathrm{CT}$, and there were probably several patients who underwent facial CT only. Thus, caution is needed when generalizing our findings. Third, only axial brain CT was performed in both study centers. Three-dimensional reconstruction of brain CT images would have increased sensitivity and specificity for detection of facial fractures. Further prospective and larger studies including three-dimensional image reconstruction are necessary.

In conclusion, the results of our study suggest that brain CT has high diagnostic value in detection of facial fractures and can aid emergency physicians when determining the requirement for additional facial CT. We recommend that when patients present with upper facial injuries, particularly those above the inferior orbital wall, brain CT should be performed first. If additional lesions are suspected and do not appear on brain CT, facial CT should be performed. Such key decisions can minimize both radiation exposure and procedure-related costs in patients with blunt head trauma.

\section{CONFLICT OF INTEREST}

No potential conflict of interest relevant to this article was reported.

\section{REFERENCES}

1. Aksoy E, Unlu E, Sensoz O. A retrospective study on epidemiology and treatment of maxillofacial fractures. J Craniofac Surg 2002;13:772-5.

2. Holmgren EP, Dierks EJ, Assael LA, Bell RB, Potter BE. Facial soft tissue injuries as an aid to ordering a combination head and facial computed tomography in trauma patients. J Oral Maxillofac Surg 2005;63:651-4.

3. Sun JK, LeMay DR. Imaging of facial trauma. Neuroimaging Clin N Am 2002;12:295-309.

4. Haug RH, Prather J, Indresano AT. An epidemiologic survey of facial fractures and concomitant injuries. J Oral Maxillofac Surg 1990;48:926-32.

5. Huang LK, Wang HH, Tu HF, Fu CY. Simultaneous head and facial computed tomography scans for assessing facial fractures in patients with traumatic brain injury. Injury 2017;48: 1417-22.

6. Marinaro J, Crandall CS, Doezema D. Computed tomography of the head as a screening examination for facial fractures. Am J Emerg Med 2007;25:616-9.

7. Lewandowski RJ, Rhodes CA, McCarroll K, Hefner L. Role of routine nonenhanced head computed tomography scan in excluding orbital, maxillary, or zygomatic fractures secondary to blunt head trauma. Emerg Radiol 2004;10:173-5.

8. Steidler NE, Cook RM, Reade PC. Incidence and management of major middle third facial fractures at the Royal Melbourne Hospital: a retrospective study. Int J Oral Surg 1980;9:92-8.

9. Whitaker LA, Yaremchuk MJ. Secondary reconstruction of posttraumatic orbital deformities. Ann Plast Surg 1990;25:440-9.

10. Wall BF, Hart D. Revised radiation doses for typical X-ray examinations: report on a recent review of doses to patients from medical X-ray examinations in the UK by NRPB. National Radiological Protection Board. Br J Radiol 1997;70:437-9.

11. Chen TW, Ng SY, Whaites EJ. Interpretation of skull radiographs for facial fractures by medical staff working in UK emergency departments: a pilot study. Dentomaxillofac Radiol 2003;32: 166-72.

12. Tanrikulu R, Erol B. Comparison of computed tomography with conventional radiography for midfacial fractures. Dentomaxillofac Radiol 2001;30:141-6.

13. Finkle $D R$, Ringler $S L$, Luttenton $C R$, Beernink JH, Peterson NT, Dean RE. Comparison of the diagnostic methods used in maxillofacial trauma. Plast Reconstr Surg 1985;75:32-41.

14. Bajwa SJ, Kaur J, Singh A, Kapoor V, Bindra GS, Ghai GS. Clinical and critical care concerns of cranio-facial trauma: a retrospective study in a tertiary care institute. Natl J Maxillofac Surg 2012;3:133-8.

15. Linnau KF, Stanley RB Jr, Hallam DK, Gross JA, Mann FA. Imaging of high-energy midfacial trauma: what the surgeon needs to know. Eur J Radiol 2003;48:17-32.

16. National Research Council. Health risks from exposure to low levels of ionizing radiation. Washington, DC: National Academies Press; 2005.

17. Brenner DJ, Hall EJ. Computed tomography: an increasing source of radiation exposure. N Engl J Med 2007;357:2277-84.

18. McNitt-Gray MF. AAPM/RSNA physics tutorial for residents ation 1985. Washington, DC: National Academies Press; 2005.

19. IMV. IMV 2006 CT market summary report. Des Plains, IL: IMV 
Medical Information Division; 2006.

20. Kaups KL, Davis JW, Parks SN. Routinely repeated computed tomography after blunt head trauma: does it benefit patients? J Trauma 2004:56:475-80.

21. Brenner DJ, Doll R, Goodhead DT, et al. Cancer risks attributable to low doses of ionizing radiation: assessing what we re- ally know. Proc Natl Acad Sci U S A 2003;100:13761-6.

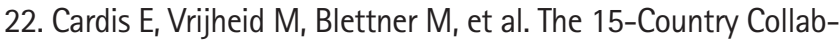
orative Study of Cancer Risk among Radiation Workers in the Nuclear Industry: estimates of radiation-related cancer risks. Radiat Res 2007;167:396-416. 